International Journal of Modern Physics A

(C) World Scientific Publishing Company

\title{
FLAVOR ASYMMETRY OF NUCLEON SEA FROM DETAILED BALANCE*
}

\author{
YONG-JUN ZHANG, BO-QIANG MA and LI-MING YANG \\ Department of Physics, Peking University, Beijing 100871, China
}

\begin{abstract}
In this study, the proton is taken as an ensemble of quark-gluon Fock states. Using the principle of detailed balance, we find $\bar{d}-\bar{u} \approx 0.124$, which is in surprisingly agreement with the experimental observation.
\end{abstract}

\section{Hypothesis of Ensemble}

In this talk, we introduce a new work 1 on the flavor asymmetry of the nucleon sea as arising from the pure statistical effect without any parameter. It will be shown that we can reproduce the flavor asymmetry $\bar{d}-\bar{u} \approx 0.123$, which is in surprisingly agreement with the experimental observation, by just using the principle of detailed balance. We take the proton as an ensemble of a complete set of quark-gluon Fock states, and these different Fock states can be written as

$$
\begin{aligned}
\left|\psi^{1}\right\rangle & =|u u d\rangle=|\{u u d\},\{0,0,0\}\rangle \\
\left|\psi^{2}\right\rangle & =|u u d g\rangle=|\{u u d\},\{0,0,1\}\rangle \\
\left|\psi^{3}\right\rangle & =|u u d \bar{u} u\rangle=|\{u u d\},\{1,0,0\}\rangle \\
\cdots & \\
\left|\psi^{n}\right\rangle & =\mid u u d \underbrace{\bar{u} u \cdots \bar{u} u}_{i} \underbrace{\bar{d} d \cdots \bar{d} d}_{j} \underbrace{g \cdots g}_{k}\}\rangle=|\{u u d\},\{i, j, k\}\rangle,
\end{aligned}
$$

where $\{u u d\}$ represents the valence quarks of the proton, $i$ is the number of quarkantiquark $u \bar{u}$ pairs, $j$ is the number of quark-antiquark $d \bar{d}$ pairs, and $k$ is the number of gluons. Then the density operator of the ensemble is

$$
\begin{aligned}
\hat{\rho} & =\rho_{0,0,0}|u u d\rangle\left\langle u u d\left|+\rho_{0,0,1}\right| u u d g\right\rangle\langle u u d g|+\cdots \\
& =\sum_{i, j, k} \rho_{i, j, k}|\{u u d\},\{i, j, k\}\rangle\langle\{u u d\},\{i, j, k\}|,
\end{aligned}
$$

*Talk presented at the Third Circum-Pan-Pacific Symposium on "High Energy Spin Physics", Oct. 8-13, 2001, Beijing, China. This work is partially supported by the National Natural Science Foundation of China. 
where $\rho_{i, j, k}$ is the probability of finding a proton in the state $|\{u u d\},\{i, j, k\}\rangle$ and should satisfy the normalization condition,

$$
\sum_{i, j, k} \rho_{i, j, k}=1 .
$$

It will be shown that $\rho_{i, j, k}$ can be calculated by using the principle of detailed balance without any parameter.

\section{The Principle of Detailed Balance}

Given an ensemble of quark-gluon Fock states that has $N_{\mathrm{A}}\left(N_{\mathrm{A}}=N \rho_{\mathrm{A}}\right)$ of finding proton in state $\mathrm{A}$ and $N_{\mathrm{B}}\left(N_{\mathrm{A}}=N \rho_{\mathrm{A}}\right)$ of finding proton in state $\mathrm{B}$, then the states may change between each others from time to time. The principle of detailed balance means that the changing between any two states balance each other. So during a period of time, the number of events that changed from state $\mathrm{A}$ to state $\mathrm{B}$ $\left(n_{\mathrm{A} \rightarrow \mathrm{B}}\right)$ equals the number of events that changed from state $\mathrm{B}$ to state $\mathrm{A}\left(n_{B \rightarrow A}\right)$,

$$
n_{\mathrm{A} \rightarrow \mathrm{B}}=n_{\mathrm{B} \rightarrow \mathrm{A}} \text {. }
$$

$n_{\mathrm{A} \rightarrow \mathrm{B}}$ is proportional to both $N_{\mathrm{A}}$ and the transition probability of $\mathrm{A}$ to $\mathrm{B}\left(R_{\mathrm{A} \rightarrow \mathrm{B}}\right)$,

$$
n_{\mathrm{A} \rightarrow \mathrm{B}}=N_{\mathrm{A}} R_{\mathrm{A} \rightarrow \mathrm{B}}=N \rho_{\mathrm{A}} R_{\mathrm{A} \rightarrow \mathrm{B}} .
$$

We considered about $\mathrm{B}$ to $\mathrm{A}$ in the same way,

$$
n_{\mathrm{B} \rightarrow \mathrm{A}}=N_{\mathrm{B}} R_{\mathrm{B} \rightarrow \mathrm{A}}=N \rho_{\mathrm{B}} R_{\mathrm{B} \rightarrow \mathrm{A}} .
$$

Then combining (9), (10) and (11), we have

$$
\frac{\rho_{\mathrm{A}}}{\rho_{\mathrm{B}}}=\frac{R_{\mathrm{B} \rightarrow \mathrm{A}}}{R_{\mathrm{A} \rightarrow \mathrm{B}}} .
$$

In order to know $\rho_{i, j, k}$, transition probabilities should be calculated out first.

The transition between two states has two ways: splitting and recombination.

In the splitting process, the transition probability is proportional to the number of partons that may split in a certain state,

$$
R_{q \Rightarrow q g} \propto N_{q}, \quad R_{g \Rightarrow \bar{q} q} \propto N_{g} .
$$

In the recombination process that involving two kinds of partons, the transition probability is proportional to both the number of those two kinds of partons that may recombine in a certain state,

$$
R_{q g \Rightarrow q} \propto N_{q} N_{g}, \quad R_{\bar{q} q \Rightarrow g} \propto N_{\bar{q}} N_{q} .
$$

For a simple illustration, we neglect the $g \Leftrightarrow g g$ processes because they give small contribution to the flavor asymmetrye.

We will use symbol

$$
\underset{R_{\mathrm{B} \rightarrow \mathrm{A}}}{\stackrel{R_{\mathrm{A} \rightarrow \mathrm{B}}}{\rightleftharpoons}}|B\rangle
$$


to present our calculations about transition probabilities.

(1) In the translation process involving the creation or annihilation of gluon, only $q \Leftrightarrow q g$ will be considered while $g \Leftrightarrow g g$ neglected. So we have

$$
|\{u u d\},\{i, j, k-1\}\rangle \underset{(3+2 i+2 j) k}{\rightleftharpoons}|\{u u d\},\{i, j, k\}\rangle .
$$

Using formula (12), we obtain a recursion formula,

$$
\frac{\rho_{i, j, k}}{\rho_{i, j, k-1}}=\frac{1}{k}
$$

We can further get a more general formula,

$$
\frac{\rho_{i, j, k}}{\rho_{i, j, 0}}=\frac{1}{k !},
$$

and we have $\rho_{i, j, 1}=\rho_{i, j, 0}$, which we will used later.

(2) In the translation process involving the creation or annihilation of a pair of $\bar{u} u$, we have

$$
|\{u u d\},\{i-1, j, 1\}\rangle \underset{i(i+2)}{\stackrel{1}{\rightleftharpoons}}|\{u u d\},\{i, j, 0\}\rangle .
$$

Using formula (12), we obtain a recursion formula,

$$
\frac{\rho_{i, j, 0}}{\rho_{i-1, j, 1}}=\frac{1}{i(i+2)} \text {. }
$$

Using relation $\rho_{i, j, 1}=\rho_{i, j, 0}$, we get

$$
\begin{gathered}
\frac{\rho_{i, j, 0}}{\rho_{i-1, j, 0}}=\frac{1}{i(i+2)}, \\
\frac{\rho_{i, j, 0}}{\rho_{0, j, 0}}=\frac{2}{i !(i+2) !} .
\end{gathered}
$$

(3) In the translation process involving the creation or annihilation of a pair of $\bar{d} d$, we have

$$
|\{u u d\},\{i, j-1,1\}\rangle \underset{j(j+1)}{\stackrel{1}{\rightleftharpoons}}|\{u u d\},\{i, j, 0\}\rangle .
$$

Using formula (12), we obtain a recursion formula,

$$
\frac{\rho_{i, j, 0}}{\rho_{i, j-1,1}}=\frac{1}{j(j+1)}
$$

Using relation $\rho_{i, j, 1}=\rho_{i, j, 0}$, we get

$$
\begin{aligned}
\frac{\rho_{i, j, 0}}{\rho_{i, j-1,0}} & =\frac{1}{j(j+1)}, \\
\frac{\rho_{i, j, 0}}{\rho_{i, 0,0}} & =\frac{1}{j !(j+1) !} .
\end{aligned}
$$


Combining (18), (22), and (26), we obtain the general formula

$$
\frac{\rho_{i, j, k}}{\rho_{0,0,0}}=\frac{2}{i !(i+2) ! j !(j+1) ! k !} .
$$

From this result and the normalization condition (8), all $\rho_{i, j, k}$ can be calculated out as shown in Table 1. From Table 1, we get the numbers of intrinsic gluons and sea quarks of the proton,

$$
\begin{aligned}
\bar{u} & =\sum_{i, j, k} i \rho_{i, j, k}=0.308 \\
\bar{d}=\sum_{i, j, k} j \rho_{i, j, k} & =0.432, \\
g=\sum_{i, j, k} k \rho_{i, j, k} & =0.997, \\
\bar{d}-\bar{u} & =0.124 .
\end{aligned}
$$

The flavor sea-quark asymmetry $\bar{d}-\bar{u}$ can be checked by experiments directly because its $Q^{2}$ dependence is small. It is a surprise that our result is in excellent agreement with the recent experimental result $\bar{d}-\bar{u}=0.118 \pm 0.012$ 且. This good agreement indicates that the principle of detailed balance plays an essential role in

\begin{tabular}{|c|c|c|c|c|c|c|c|c|}
\hline $\bar{i}$ & $\mathrm{j}$ & $|\{u u d\},\{i, j, 0\}\rangle$ & $\rho_{i, j, 0}$ & $\rho_{i, j, 1}$ & $\rho_{i, j, 2}$ & $\rho_{i, j, 3}$ & $\rho_{i, j, 4}$ & $\cdots$ \\
\hline 0 & 0 & $|u u d\rangle$ & 0.167849 & 0.167849 & 0.083924 & 0.027975 & 0.006994 & $\cdots$ \\
\hline 1 & 0 & $|u u d \bar{u} u\rangle$ & 0.055950 & 0.055950 & 0.027975 & 0.009325 & 0.002331 & $\ldots$ \\
\hline 0 & 1 & $|u u d \bar{d} d\rangle$ & 0.083924 & 0.083924 & 0.041962 & 0.013987 & 0.003497 & $\cdots$ \\
\hline 1 & 1 & $|u u d \bar{u} u \bar{d} d\rangle$ & 0.027975 & 0.027975 & 0.013987 & 0.004662 & 0.001166 & $\ldots$ \\
\hline 0 & 2 & $|u u d \bar{d} d \bar{d} d\rangle$ & 0.013987 & 0.013987 & 0.006994 & 0.002331 & 0.000583 & $\ldots$ \\
\hline 2 & 0 & $|u u d \bar{u} u \bar{u} u\rangle$ & 0.006994 & 0.006994 & 0.003497 & 0.001166 & 0.000291 & $\ldots$ \\
\hline 1 & 2 & $|u u d \bar{u} u \bar{d} d \bar{d} d\rangle$ & 0.004662 & 0.004662 & 0.002331 & 0.000777 & 0.000194 & $\ldots$ \\
\hline 2 & 1 & $|u u d \bar{u} u \bar{u} u \bar{d} d\rangle$ & 0.003497 & 0.003497 & 0.001748 & 0.000583 & 0.000146 & $\ldots$ \\
\hline 0 & 3 & $|u u d \bar{d} d \bar{d} d \bar{d} d\rangle$ & 0.001166 & 0.001166 & 0.000583 & 0.000194 & 0.000049 & $\ldots$ \\
\hline 3 & 0 & $|u u d \bar{u} u \bar{u} u \bar{u} u\rangle$ & 0.000466 & 0.000466 & 0.000233 & 0.000078 & 0.000019 & $\ldots$ \\
\hline$\cdots$ & $\cdots$ & $\ldots$ & $\cdots$ & $\cdots$ & $\cdots$ & $\cdots$ & $\cdots$ & $\cdots$ \\
\hline
\end{tabular}
the structure of proton. We also give a complete set of Fock states for the proton, with the probability of finding each Fock state calculated without any parameter, as shown in Table 1.

Table 1. The probabilities, $\rho_{i, j, k}$, of finding the quark-gluon Fock states of the proton.

\section{References}

1. Y.-J. Zhang, B. Zhang and B.-Q. Ma, Phys. Lett. B523, 260 (2001). There is one misprint in formula (11), in which " 3 " should be "5".

2. FNAL E866/NuSea Collaboration, R.S. Towell et al., Phys. Rev. D64, 052002 (2001). For a recent review, see, e.g., G. T. Garvey and J. C. Peng, Prog. Part. Nucl. Phys. 47, 203 (2001). 